IRA-International Journal of Technology \& Engineering ISSN 2455-4480

Proceedings of the

International Conference on Science \& Engineering for Sustainable Development (2017)

Pg. no.279-285

Published by: Institute of Research Advances https://research-advances.org/index.php/IRAJTE

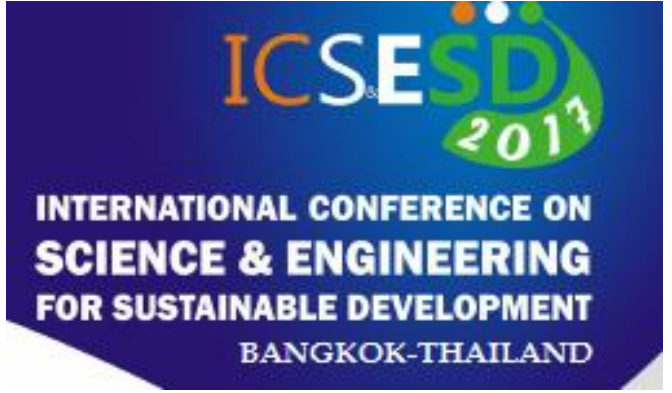

\title{
Three Dimensional MR Brain Image Reconstruction and Interactive Segmentation Based Tumor Region Extraction Using Active Contour Models
}

\author{
Nikhil Gala* ${ }^{1}$, K.D. Desai ${ }^{2}$ \\ ${ }^{1}$ Assistant Professor, Department of Electronics \& Telecommunication, NMIMS \\ University's Mukesh Patel School of Technology Management \& Engineering, Mumbai, \\ Maharashtra, India. \\ ${ }^{2}$ Academic and Technical Advisor, Guru Gobind Singh college of Engineering \& \\ Research Centre, Nashik, Maharashtra, India.
}

Type of Review: Originality Check \& Peer Review under the responsibility of the Scientific Committee of the Conference and The Institution of Engineers (India). DOI: http://dx.doi.org/10.21013/jte.ICSESD201727

How to cite this paper:

Gala, N., Desai, K. (2017). Three Dimensional MR Brain Image Reconstruction and Interactive Segmentation Based Tumor Region Extraction Using Active Contour Models. Proceedings of the International Conference on Science \& Engineering for Sustainable Development (2017), 279285. doi: http://dx.doi.org/10.21013/jte.ICSESD201727

(C) International Conference on Science \& Engineering for Sustainable Development\& The Institution of Engineers (India).

\section{(cc) BY-NC}

This work is licensed under a Creative Commons Attribution-Non Commercial 4.0 International License subject to proper citation to the publication source of the work.

Disclaimer: The conference papers as published by the Institute of Research Advances (IRA) are the views and opinions of their respective authors and are not the views or opinions of the IRA. The IRA disclaims of any harm or loss caused due to the published content to any party. 


\begin{abstract}
Magnetic resonance imaging (MRI) system projects the anatomical structures of brain onto spatial domain. Three-dimensional (3D) construction of brain tumor using several slides of MRI has always been of keen interest for diagnosis and for research purpose. This paper presents a three dimensional brain image reconstruction methodology which is very much helpful in predicting the depth of any abnormality in the image and also proposes an active contour based approach for the extraction of the abnormal (tumor) region. The extracted regions are modeled using Iso surface $3 D$ view models.
\end{abstract}

\title{
Introduction
}

MRI is a popularly known imaging modality and is used as the best neuro-imaging tool for evaluating and following up the examination of the patients for numerous causes. It is beneficial due to its high contrast resolution and prevention from using the ionizing radiations. The particular medical imaging technique is also not preferable for the detection of the small lesions and iso-dense lesions. Among its several benefits, MRI technique is being selected in this study due to its ability of generating images in the Sagital, axial, and coronal planes. This can also provide help in improved localization of the lesion within the 3D space, eventually allowing structures to become more explicitly delineated.

The most challenging task in modern medical imaging systems is to detect the abnormality in the acquired MRI brain images and the reason behind the abnormalities detection failure is due to the complex structure of brain and it needs different convergence techniques which act in very high speed [1].

The two step process implemented for detection of MRI image is as follows

(i) Classification algorithms have tremendously increased in past two decades and the brain image abnormalities detection is classified by using the reputed classification approach and the resultant classified based on various parameters to differentiate between the normal and abnormal [2],[3].

(ii) The volumetric analysis is carried into latter step on the segmented data which is acquired from previous step i.e. classification approach. The entire process is automatic consumes less time and achieves more performance compare to traditional approaches [4].3D-visualization is a technique for 3D imaging from 2D images. The sequence of 2D MR slices cannot convey the exact complexity of human brain abnormality, so extensive training has to be done for the radiologists to interpret it. So there is a need to visualize this anatomy in 3D by interpolating the $2 \mathrm{D}$ slices. 3Dview enables a better understanding of the topology, shape and depth of the tumor which is very much helpful in research and surgical planning. However these interpolated slices may lack of some information, so reconstruction of this $3 \mathrm{D}$ view by a suitable surface algorithm is of great importance [5], [6].

This paper presents a methodology for 3D image reconstruction and extraction of tumor region. This paper is organized as follows, section I presents the introduction and importance of the research, section II presents the literature survey on the latest research and trends in 3D visualization, section III presents the methodology that is followed in this context, section IV presents the experimental results that were obtained using the standard database images slice set, ending up with the conclusions.

\section{Related work}

3D image segmentation is one of the important problems in medical image processing, in general extraction of the abnormal portion of the brain MR image and projecting it in 3D space is of great importance. In this section a brief literature survey of the related work that has been developed so far is presented Vijay and Subhashini in 2014 [7] has discussed that the Segmentation of images holds an 
important position in the region of image processing. It becomes more important while typically working with medical images where pre-surgery and post-surgery decisions are needed for the objective of initiating and speeding up the recovery process. Computer aided detection of abnormal growth of tissues is primarily motivated by the necessity of achieving maximum possible accuracy. Manual segmentation of those abnormal tissues can't be in contrast to modern day's high speed computing machines which enable us to visually observe the quantity and location of unwanted tissues.Solmaz Abbasi and Farshad Tajeri Pour in [8] presents the previous techniques for image segmentation to find the brain tumor are of 2-Dimension. Now in this paper 3-Dimentional medical image segmentation is present. In this technique to identify the brain tumor in MRI images clustering and classification techniques are performed. By this technique we can reduce the time and memory. The classification can be done with the criteria of dice's and jacquard's coefficient on the brain tumor from MRI images retrieved from the Brats 2013 database. Heena Hooda et al in [9] presents the previous techniques of image segmentation are K-means clustering, Fuzzy C-means Clustering and Region Growing for detection of brain tumor from MRI images. The performance can be calculated by analyzing error percentage associated to ground to ground truth. By performing this task exact location, orientation and abnormal area of tissues can be identified.Tao Wang et.al in [10], presented the segmentation is done by using the methodology Traditional Snake, GVF (gradient vector flow) Snake, BVF Snake, and Magneto static Active Counter (MAC). GVF Snake method is to find the boundaries of the brain tumor. BVK snake will improve capture range and concave object extraction capability. All these method are mainly used for the extraction of brain tumors. The various fluid vector flow (FVF) techniques are boundary vector flow, gradient vector flow, and MAC. The MAC will contain three set of experiments those are (1) synthetic images (2) pediatric set MRI images (3) brain tumor MRI images. FVF is not suitable for analyzing 3-D medical data. The further process of an image can be done by interpolation process. This paper has also adopted an interactive approach based tumor segmentation using GVF method.

\section{D MR Image Reconstruction}

\section{(A) Interactive GVF Approach}

Original Snake algorithm was introduced by Kass et.al in [11] in which the contour deforms to minimize the contour energy that includes the internal energy from the contour and the external image of the image. The short comings of the original snake model was clearly discussed in [12] by Xu et.al . The external force field for the original and distance snake is ir-rotational and based on the contour points and the closest edge points in contour points in normal direction. This limits the deformation unto boundary concavities because there is no external force pointing inside. GVF based snake achieves these limitations and in addition the magnitude if the external force are the same over the whole image.

The GVF snake has the external force represented as

$\mathrm{F}(\mathrm{v})=(\alpha(\mathrm{x}, \mathrm{y}), \beta(\mathrm{x}, \mathrm{y}))$ and $\mathrm{F}(\mathrm{v})$ can be obtained by minimizing the energy functional

$\mathrm{E}=\iint \mu\left(\alpha_{\mathrm{x}}^{2}+\alpha_{\mathrm{y}}^{2}+\beta_{\mathrm{x}}^{2}+\beta_{\mathrm{y}}^{2}\right)+|\nabla \mathrm{f}|^{2}|\mathrm{~F}-\nabla \mathrm{f}|^{2} \mathrm{dxdy}$

Where ' $\mathrm{f}$ ' is an edge map of the input image $\mathrm{I}$, and $\mu$ is a regularization parameter. Using the variational method, F can be found by solving the following the Euler Equation

$\left\{\begin{array}{l}\mu \nabla^{2} \alpha-\left(\alpha-\mathrm{f}_{\mathrm{x}}\right)\left(\mathrm{f}_{\mathrm{x}}^{2}+\mathrm{f}_{\mathrm{y}}^{2}\right)=0 \\ \mu \nabla^{2} \beta-\left(\beta-\mathrm{f}_{\mathrm{y}}\right)\left(\mathrm{f}_{\mathrm{x}}^{2}+\mathrm{f}_{\mathrm{y}}^{2}\right)=0\end{array}\right.$

This approach is an interactive approach where the user intervention is required for selecting the region of interest. 


\section{(B) 3D Visualization}

In this work a database of MRI dataset from BRATS MR image database with 129 slices for each subject from Cancer imaging Archive are used. From this dataset those slices which do contain the abnormal region are chosen for the segmentation process. Obviously, having more slices lead us to reconstruct a clearer and more reliable 3D tumor. Due to limited number of slices in MRI scans, predicted intermediate slices from the real slices using interpolation is proposed to increase the number of slices to improve reconstruction. Generally, the set of slices acquired from the MRI device is such that the distance between the slices is larger than the distance between the pixels within the slice. The surface reconstructed with such a set of slices is inaccurate and not smooth. The original shape based interpolation proposed by Raya et al in [13] converts binary image into a gray scale image via a city block distance transform which assigns to every point in the binary image a gray level equal to its shortest distance from the boundary of the object. Then the intermediate slice is estimated by linearly interpolating the distances on two sequential slices.

Finally, the interpolated image is converted to a binary image by thresholding at zero. The Euclidian distance transform is a global operation since for each pixel a shortest distance to boundary pixel must be assigned. Hence, the city block distance transform which computes the distance transform based on nneighborhood was used in the original shape based interpolation. However, the city block distance metric provides faster computation of the distance transform but it provides a bad approximation to the Euclidian distance. This is because, the city block distance metric over estimates the diagonal distances since it counts diagonal connections as 2 steps, rather than $\sqrt{2}$. Another problem with shape based interpolation is that it produces anomalous results if there is a drastic shift in the object position on sequential slices. That is the object reconstructed with such a set of slices is not accurate. In order to overcome this problem we perform centroid alignment of the tumors to match the centroids of tumors in the consecutive slices prior to interpolation. This results in gradual changes in both shapes and spatial positions of the object in interpolated slices.

\section{Proposed Approach}

In this paper the interactive based segmentation for the abnormal images is performed and finally the 3D volumetric visualization is presented. The proposed approach is done in the following steps

- Read the DICOM image from standard BRATS MR image dataset available at [15]

- Select the slices for abnormal region extraction

- Apply GVF based interactive approach of image segmentation to extract the tumor region

- Interpolated the 2D slices of extracted regions and render them

- Using Iso surface models project the rendered slices in $3 \mathrm{D}$ view

The pictorial description is depicted below, during the segmentation process of the abnormal region for selected slice, the initial region of interest of the first slice is given by user interaction which is automated for the rest of the slices. Thus, the abnormal region position is obtained using the GVF model. The results which are obtained using the above are tabulated below in step wise manner. 

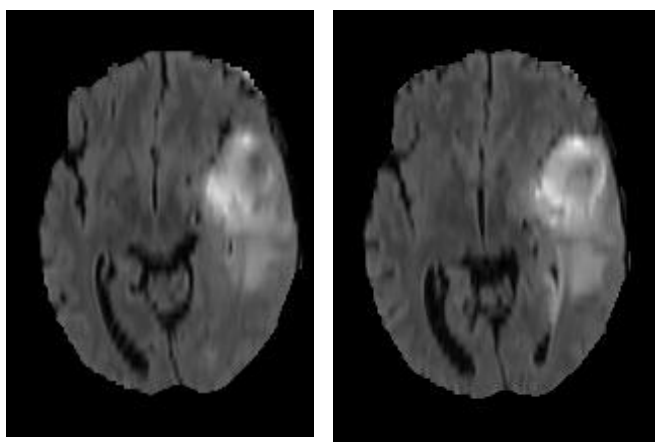

(a)

(b)
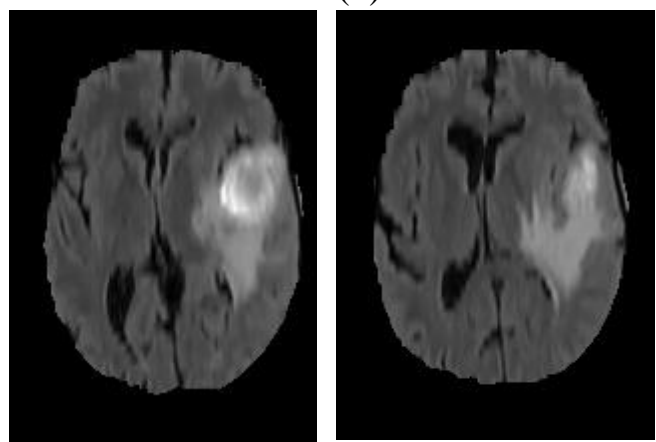

(c)

(d)

Figure 1: (a) Slice 60 (b) slice 64 (c) slice 68 (d) slice 72

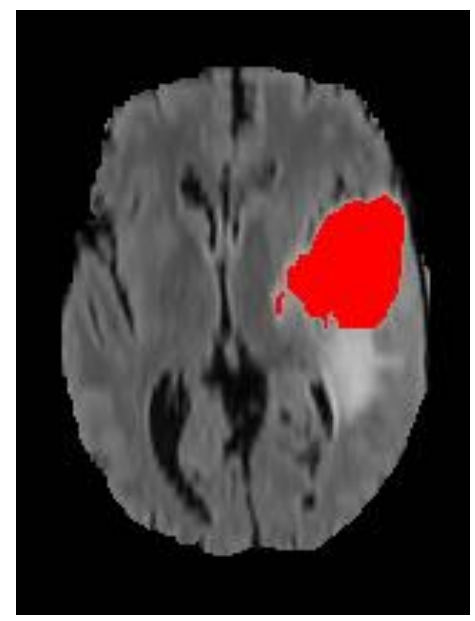

Figure 2: Detection of Abnormal Region 


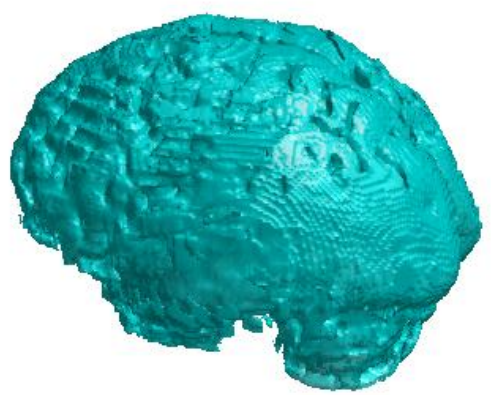

Figure 3: 3D-View of Brain Image using Iso surface model

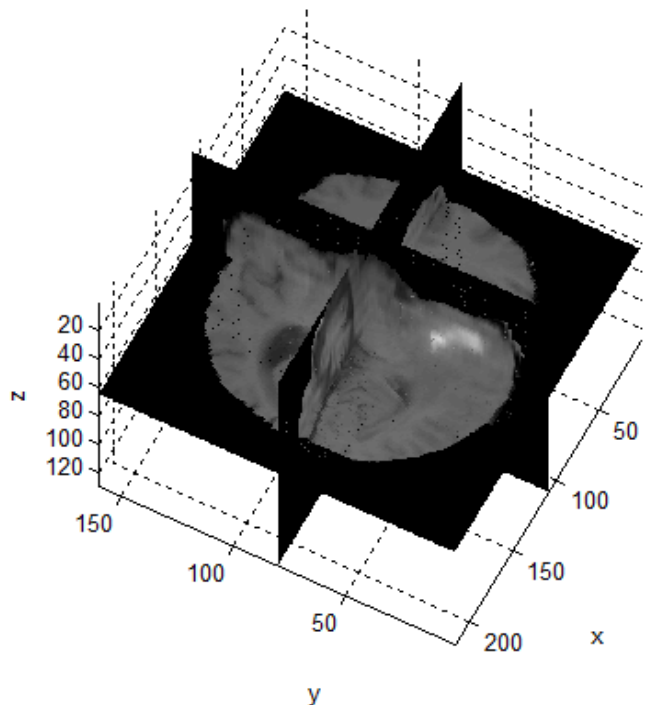

Figure 4: Slico-metric view of the slices

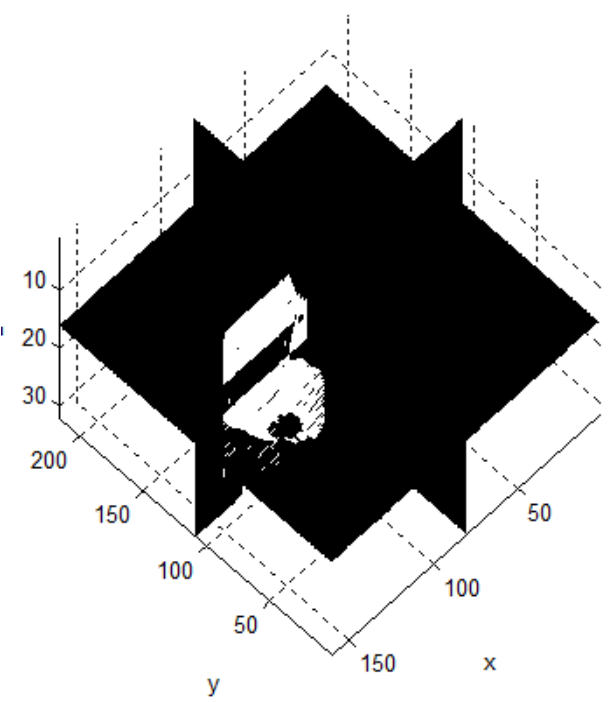

Figure 5: Slico-Metric view of abnormal regions in Slices 


\section{Conclusion}

Image segmentation and 3D visualization is presented in this paper, where the tumor region positions are noted and extracted using the GVF based snake model which is 3D modeled using the Iso surface models. The proposed method is tested for more than 10 BRATS MR images and found that the method is providing the satisfactory results. The method can be further extended by incorporating it with hybrid approaches of segmentations algorithm to increase the segmentation efficiency and also to include the performance evaluation metric for the assessment of segmentation process. Volume estimation of the abnormal region can also be included.

\section{References}

[1] Pratik P. Singhai, Siddharth A. Ladhake, "Brain Tumor Detection Using Marker Based Watershed Segmentation from Digital MR Images”. IJITEE, ISSN 2278-3075, Volume (2), Issue (5), April 2013.

[2] M. Gouskir, M. Boutalline, B. Bouikhalene, H. Aissaoui, B. Elhadadi, "Automatic Brain Tumor Extraction for MRI-T1 and T2 using Geodesic Distance and Statistical Methods". IJARCSSE, ISSN 2277 128X, Volume (3), Issue (12), December 2013.

[3] Vinay Parameshwarappa, Nandish S, "A Segmented Morphological Approach to Detect Tumor in Brain Images”. IJARCSSE, ISSN 2277 128X, Volume (4), Issue (1), January 2014.

[4] Atiq Islam, Syed M. S. Reza, and Khan M. Iftekharuddin , "Multifractal Texture Estimation for Detection and Segmentation of Brain Tumors" IEEE Transactions On Biomedical Engineering, Vol. 60, No. 11, November 2013, ISSN- 0018-9294.

[5] K. Narayan and Y. Karunakar, "3-D Reconstruction of Tumors in MRI Images”, International Journal of Research and Reviews in Signal Acquisition and Processing, vol. 2, no. 1,2011

[6] N. Archip, R. Rohling, V. Dessenne, P. J. Erard and L. P. Nolte, "Anatomical structure modeling from medical images", Computer Methods and Programs in Biomedicine, vol. 82,2006.

[7] Vijay and Subhashini. "Performance Analysis of Fuzzy C Means Algorithm in Automated Detection of Brain Tumor." Computing and Communication Technologies (WCCCT), 2014 World Congress , IEEE, 2014

[8] S. Abbasi and F. Tajeri Pour, "A hybrid approach for detection of brain tumor in MRI images," 2014 21th Iranian Conference on Biomedical Engineering (ICBME), Tehran, 2014, pp. 269-274

[9] Hooda, H, Verma, O. P., \& Singhal, T. (2014, May). Brain tumor segmentation: A performance analysis using K-Means, Fuzzy C-Means and Region growing algorithm. In Advanced Communication Control and Computing Technologies (ICACCCT), 2014 International Conference on (pp. 1621-1626).

[10] T. Wang, I. Cheng and A. Basu , "Fluid Vector Flow and Applications in Brain Tumor Segmentation," in IEEE Transactions on Biomedical Engineering, vol. 56, no. 3, pp. 781-789, March 2009.

[11] M. Kass, A. Witkin, D. Terzopoulos, "Snakes: active contour models", International Journal of Computer. Vision. 1 (4) (1988) 321-331

[12] C. Xu, J. Prince, "Snakes, shapes, and gradient vector flow", IEEE Trans. Image Process. 7 (3) (1998) 359-369

[13] S. P. Raya and J. K. Udupa, "Shape based interpolation of multidimensional objects", IEEE Transaction on Medical Imaging, vol. 1, no. 9, (1990)

[14] Bosma, M. K., Smit, J., Lobregt, S.: Iso-surface volume rendering. In Kim, Y., Mun, S. K.(Eds.): Medical Imaging 1998: Image Display, Proc. SPIE 3335. San Diego, CA, 1998,10-19

[15] http://braintumorsegmentation.org/ 